\title{
The Scheduling and Energy Complexity of Strong Connectivity in Ultra-Wideband Networks
}

\author{
Qiang-Sheng Hua \\ Department of Computer Science \\ The University of Hong Kong \\ Pokfulam Road, Hong Kong, P.R. China \\ qshua@cs.hku.hk
}

\author{
Francis C.M. Lau \\ Department of Computer Science \\ The University of Hong Kong \\ Pokfulam Road, Hong Kong, P.R. China \\ fcmlau@cs.hku.hk
}

\begin{abstract}
Recently Moscibroda and Wattenhofer came up with the notion of scheduling complexity to capture the minimum amount of time to successfully schedule all the transmission requests under the physical SINR model. Their algorithm featuring a nonlinear power assignment can schedule strongly connected transmissions in narrowband networks with $O\left(\log ^{4} n\right)$ timeslots. In this paper, we first generalize this result to ultra-wideband networks. We show the strong connectivity scheduling complexity in UWB networks to be $O\left(\log (n / m) \cdot \log ^{3} n\right)$, where $m$ is the processing gain. Secondly, we show that both of these polylogarithmic scheduling complexity results are gained at the expense of exponential energy complexity with lower bound $\omega\left(n \cdot 2^{n}\right)$. We also prove the upper bound of the energy complexity in narrowband networks to be $O\left(n^{2} \cdot 2^{n \alpha}\right)$, and for UWB networks, this upper bound can be reduced by a processing gain factor.

On the other hand, we show that improving the scheduling complexity through arbitrary power control has its limitations, and that different power assignment strategies have different impacts on the protocol interference models, which was often neglected in the design of wireless scheduling algorithms. Compared with narrowband networks, although the effect of aggregate interferences in UWB networks is greatly reduced, we demonstrate that the constant and linear power assignments in UWB networks are still inefficient in the worst case with respect to the scheduling complexity $(\Omega(n / m))$, which suggests there is a need for a better arbitrary power assignment.

Our analyses shed new light on the design of the power assignment scheme and the performance analysis of the wireless scheduling algorithms. In energy-constrained wireless networks, a tradeoff between the scheduling complexity and energy complexity is a practical consideration. Our results in this paper can be directly applied to other spread-spectrum networks including DS-CDMA and FH-CDMA.
\end{abstract}

\section{Categories and Subject Descriptors}

C.2.1 [Computer-Communication Networks]: Network Architecture and Design - wireless communication, network topology;

\footnotetext{
Permission to make digital or hard copies of all or part of this work for personal or classroom use is granted without fee provided that copies are not made or distributed for profit or commercial advantage and that copies bear this notice and the full citation on the first page. To copy otherwise, or republish, to post on servers or to redistribute to lists, requires prior specific permission and/or a fee.

MSWiM'06, October 2-6, 2006, Torremolinos, Malaga, Spain.

Copyright 2006 ACM 1-59593-477-4/06/0010 ...\$5.00.
}

\begin{abstract}
F.2.2 [Analysis of Algorithms and Problem Complexity]: Nonnumerical Algorithms and Problems - geometrical problems and computations, sequencing and scheduling;
\end{abstract}

\section{General Terms}

Algorithms, Theory, Performance

\section{Keywords}

Ad hoc and sensor networks, scheduling complexity, energy complexity, interference models, interference control, ultrawideband.

\section{INTRODUCTION}

The scheduling complexity problem in wireless networks is to try to use the minimum amount of time to successfully schedule all the transmission requests to meet some topological property requirement. Recently Moscibroda and Wattenhofer proposed a solution of which the scheduling complexity for narrowband networks with strong connectivity property is only $O\left(\log ^{4} n\right)$ timeslots [10], which is exponentially faster than $O(n)$ if individual transmissions are scheduled one by one. In this paper we generalize this result to cover also ultra-wideband (UWB) networks which are drawing increasing attention due to their many promising features [17]. Since a UWB network is inherently a spread-spectrum network [12], the aggregate interferences caused by other simultaneous transmissions at the intended receiver can be reduced by a factor of the processing gain, making it very competitive in wireless communications. Unlike the narrowband networks where the interference range is larger than the transmission range, as shown in Section 3, the interference range of UWB networks around the receiver is smaller than the transmission range, allowing more simultaneous transmissions at the receiver. And one of the recent findings in UWB network research [13] is that the design of the optimal MAC can be independent of the choice of routing. Thus the use of ultrawideband can re-introduce the notion of layer separation between the MAC and the routing layers just like in traditional wire line networks. This will make the resultant network more scalable and a good choice for generic sensor networks. Furthermore, UWB is multi-path fading resistant, and as the following SINR model shows, it is more flexible in terms of adapting its parameters to meet different operational requirements (e.g., change in processing gain).

For our analyses, we adopt the physical signal-to-interferenceplus-noise ratio (SINR) model, with which only when the received power is above the SINR ratio threshold can a message be successfully received. The SINR model for UWB networks was first given in [4], which is different from that for narrowband 
networks [6]. Specifically, the achieved signal-to-interferenceplus-noise ratio at the receiver of link $i$ can be represented as:

$$
\operatorname{SINR}_{i}=\frac{P_{i} / d\left(x_{i}, x_{j}\right)^{\alpha}}{R_{i}\left[\eta+T_{f} \sigma^{2} \sum_{k=1, k \neq i}^{N_{0}} P_{k} / d\left(x_{k}, x_{j}\right)^{\alpha}\right]} \geq \beta
$$

where

$P_{i}$ denotes the average transmission power of link i's transmitter, $x_{i}$;

$R_{i}$ denotes link $i$ 's data rate, and $R_{i}=1 /\left(N_{s} N_{h} T_{c}\right) ; N_{s}$ denotes the number of pulses per symbol, $N_{h}$ the number of timeslots per Pulse Repetition Interval (PRI), and $T_{c}$ the pulse duration;

$T_{f}$ is the PRI, and $T_{f}=N_{h} T_{c}$;

$\sigma^{2}$ is a parameter depending on the shape of the monocycle;

$\eta$ is the background noise plus interference from other non-UWB systems;

$d\left(x_{i}, x_{j}\right)$ denotes the Euclidean distance between transmitter $x_{i}$ and $x_{j}$;

$\alpha$ is the path loss exponent and $\beta$ is the SINR threshold;

$N_{0}$ denotes the number of simultaneous transmissions with transmitter $x_{i}$.

If we set $N=\eta /\left(T_{f} \sigma^{2}\right)$ and $m=1 /\left(R_{i} T_{f} \sigma^{2}\right)$, the above is transformed to a form similar to the spread-spectrum SINR model in [5]:

$$
\operatorname{SINR}=\frac{P_{i} / d\left(x_{i}, x_{j}\right)^{\alpha}}{N+\sum_{k=1, k \neq i}^{N_{0}} P_{k} / d\left(x_{k}, x_{j}\right)^{\alpha}} \geq \frac{\beta}{m}
$$

Here we use $m$ for the processing gain. If $m=1$, this becomes the traditional narrowband SINR model, as used in [10].

The organization of the paper is as follows. In Section 2, we review interference control techniques for both narrowband and wideband networks, and for both graph coloring based and physical SINR models. In Section 3, we explore different power assignments and their impacts on pair-wise interference models which play a very important role in the design of wireless protocols and wireless network capacity analyses. Furthermore, we discuss the limitations of spatial reuse through power control, which make the task to apply proper power control techniques to improving the scheduling complexity non-trivial. In Section 4, we give a formal definition of the scheduling complexity problem and show that both the constant and linear power assignments are inefficient with respect to the scheduling complexity in UWB networks. In Section 5, we generalize the non-linear power assignment algorithm in [10], with the guarantee that all the simultaneous transmissions can be successfully scheduled based on the SINR model, and show that the scheduling complexity of strong connectivity in UWB networks is $O\left(\log (n / m) \cdot \log ^{3} n\right)$. This result represents an improvement over that for the narrowband networks. In Section 6, we define the energy complexity of the wireless scheduling problems, and show that the polylogarithmic scheduling complexity was achieved at the expense of the exponential energy. And in UWB networks, the upper bound of the energy complexity can be reduced by a processing gain factor. Section 7 concludes the paper and discusses some future tasks that could make our algorithm practical.

To the best of our knowledge, this is the first paper to study both the scheduling complexity and energy complexity of UWB (and wideband) networks, as well as the first to give energy complexity analyses of the strong connectivity scheduling problem in narrowband networks. Furthermore, this is also the first paper to analyze the impacts of power control on the protocol interference models.

\section{RELATED WORK}

Interferences caused by concurrent transmissions in the shared communication medium could be nightmarish in wireless networks especially in multi-hop ad-hoc or sensor networks. If there are too many simultaneous transmissions, even if the interference from a single node is small, the aggregate interference could be disastrous. On the other hand, if there are only a few concurrent transmissions, or even to schedule them one by one would waste valuable bandwidth and the throughput capacity would suffer. Thus finding a proper interference control method is extremely important in wireless networks. And since interference control is based on the model used, therefore finding an appropriate interference model is equally important. For a recent survey on the algorithmic models of wireless ad-hoc and sensor networks, please refer to [14].

The early interference control technique used in packet radio networks is to avoid the so-called "primary interference" and "secondary interference" problems [15]. By primary interference, a single node cannot perform two operations at the same time, such as receiving from two senders, transmitting to two receivers, or receiving and sending at the same time. By secondary interference, node A is covered in sender B's transmitting range but the intended receiver is node $\mathrm{C}$ which is different from node A. Obviously avoiding secondary interference prevents the capture effect, which should be a good thing in the physical reality. The standard interference control technique to deal with these interferences is the "graph-based scheduling algorithm" [2]. It was claimed that the protocol interference model in [6] is its generic form. But as we will show in Section 3, the latter protocol model is indeed different from the graph-based one.

Based on the primary/secondary interference model, "distance-2 matching" (or strong edge coloring) for link scheduling and "distance- 2 coloring" for broadcast scheduling were proposed [1]. We focus on link scheduling in this paper. In distance-2 matching, only links that are at least of distance two apart can be assigned the same timeslot (or color). This matching is actually in line with the 802.11 DCF MAC protocol, where RTS/CTS virtual carrier sensing would block the links within a distance of two edges. But recent research indicates that both the physical carrier sensing and virtual carrier sensing methods in 802.11-ad-hoc are not throughput capacity-efficient [3]. So in this paper we will not consider this kind of distance-2 matching model. Instead, we focus on the primary interference problem that is, all pairs of links sharing a common endpoint will not be scheduled at the same time, and the reception must satisfy the SINR inequality (1) but no other constraints.

For the graph-based models, it was shown in [2] that the scheduling of the maximum cardinality of the independent sets of the conflict graph [8] cannot guarantee the best throughput capacity due to the aggregate interference effect as just mentioned. Therefore most of the recent research has shifted to analyzing 
physical SINR models [3][10]. For a latest survey of using physical SINR models in cellular networks, please refer to [9]. Regarding the use of SINR models in multi-hop networks, most of the interference control techniques focus on narrowband networks, and only a few of them would take on the spread-spectrum SINR model [5][18]. As we have mentioned, the transformed UWB SINR model is similar to the spread-spectrum SINR model, so all the results in this paper can be directly applied to other spreadspectrum networks, such as DS-CDMA and FH-CDMA.

\section{POWER ASSIGNMENT IMPACT ON PROTOCOL INTERFERENCE MODELS AND LIMITATIONS}

In this section, we first discuss the impact of different power assignments on the pair-wise interference models. And then we discuss the limitations of improving the spatial reuse through power control. Comparing with narrowband networks, there is more room for UWB networks to take advantage of power control to improve the scheduling complexity.

\subsection{Protocol Interference Models Under Different Power Assignments}

We focus on the impact of the power assignments on the pairwise interference models, which was often neglected in wireless scheduling algorithm design. Basically, we can divide the current power assignment strategies into three categories:

1) Constant power assignment;

2) linear power assignment ; and

3) arbitrary power assignment (e.g., non-linear power assignment). We first consider narrowband networks. According to inequality (1) and because $m=1$ in narrowband networks, in order to ensure a successful transmission $\left(x_{s}, x_{r}\right)$, the following inequality must hold.

$$
\frac{P_{x} / d\left(x_{s}, x_{r}\right)^{\alpha}}{N+P_{y} / d\left(y_{s}, x_{r}\right)^{\alpha}} \geq \beta \Rightarrow \frac{d\left(y_{s}, x_{r}\right)}{d\left(x_{s}, x_{r}\right)}>\beta^{\frac{1}{\alpha}} \cdot\left(\frac{P_{y}}{P_{x}}\right)^{\frac{1}{\alpha}}
$$

a) Protocol interference model with constant power assignment in narrowband networks:

With the constant power assignment, $P_{x}=P_{y}$, so by inequality (2), we have

$$
d\left(y_{s}, x_{r}\right)>\beta^{1 / \alpha} \cdot d\left(x_{s}, x_{r}\right)
$$

If we set $(1+\Delta)=\beta^{1 / \alpha}$, this becomes the same as the protocol model given in [6]. Since in narrowband networks, usually the threshold $\beta>1$ and consequently the range $\beta^{1 / \alpha} \cdot d\left(x_{s}, x_{r}\right)$ is greater than the sender's transmission range $d\left(x_{s}, x_{r}\right)$. Thus to ensure a successful transmission, a disc of radius at least $\beta^{1 / \alpha} \cdot d\left(x_{s}, x_{r}\right)$ around each successful receiver $x_{r}$ must not contain other transmitters. So we denote $\beta^{1 / \alpha} \cdot d\left(x_{s}, x_{r}\right)$ as the interference range (or exclusion region) around each receiver $x_{r}$. For example, in Fig. 1(a), assuming constant power assignment, since $d\left(x_{s}, y_{r}\right)<d\left(y_{s}, y_{r}\right)$, transmission $\left(y_{s}, y_{r}\right)$ is not successful; whereas, since $d\left(y_{s}, x_{r}\right)>d\left(x_{s}, x_{r}\right)$, transmission $\left(x_{s}, x_{r}\right)$ is successful. With this we can distinguish the graph-based interference model from the protocol interference model which was considered the same in [2]. Notice that the protocol interference model originates from the physical SINR model, and so it can reflect the physical reality including the "capture effect", while all the graph-based interference models cannot reflect the same. For example, since node $x_{r}$ is in the transmission range of $y_{s}$, it suffers from the secondary interference problem [15], so transmission $\left(x_{s}, x_{r}\right)$ is not successful.

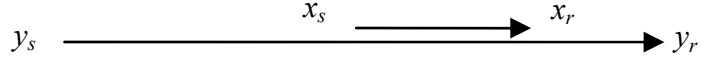

(a) $d\left(x_{s}, x_{r}\right)=1, d\left(y_{s}, y_{r}\right)=4, d\left(x_{s}, y_{r}\right)=2, d\left(y_{s}, x_{r}\right)=3$

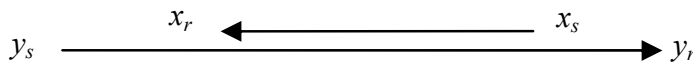

(b) $d\left(x_{s}, x_{r}\right)=2, d\left(y_{s}, y_{r}\right)=4, d\left(x_{s}, y_{r}\right)=1, d\left(y_{s}, x_{r}\right)=1$

$y_{s} \longrightarrow y_{r} \quad x_{s} \longrightarrow x_{r}$

(c) $d\left(x_{s}, x_{r}\right)=1, d\left(y_{s}, y_{r}\right)=1, d\left(x_{s}, y_{r}\right)=1, d\left(y_{s}, x_{r}\right)=3$

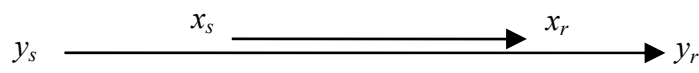

(d) $d\left(x_{s}, x_{r}\right)=2, d\left(y_{s}, y_{r}\right)=4, d\left(x_{s}, y_{r}\right)=3, d\left(y_{s}, x_{r}\right)=3$

Figure 1. Pair-wise transmissions examples

b) Protocol interference model with linear power assignment in narrowband networks:

With the linear power assignment, $P_{x}=\rho \cdot d\left(x_{s}, x_{r}\right)^{\alpha}$ and $P_{y}=\rho \cdot d\left(y_{s}, y_{r}\right)^{\alpha}$, and so according to inequality (2), we have

$\frac{d\left(y_{s}, x_{r}\right)}{d\left(x_{s}, x_{r}\right)}>\beta^{\frac{1}{\alpha}} \cdot \frac{d\left(y_{s}, y_{r}\right)}{d\left(x_{s}, x_{r}\right)} \Rightarrow d\left(y_{s}, x_{r}\right)>\beta^{\frac{1}{\alpha}} \cdot d\left(y_{s}, y_{r}\right)$

This protocol model was used in [14]. But compared with (3), it has attracted much less attention mostly because many capacity analysis papers assume the constant power assignment. Note that the interference range of receiver $x_{r}$ has been changed from $\beta^{1 / \alpha} \cdot d\left(x_{s}, x_{r}\right)$ to $\beta^{1 / \alpha} \cdot d\left(y_{s}, y_{r}\right)$. For example, in Fig. 1(a), assuming linear power assignment, since $d\left(y_{s}, x_{r}\right)<d\left(y_{s}, y_{r}\right)$, transmission $\left(x_{s}, x_{r}\right)$ is not successful. And since $d\left(x_{s}, y_{r}\right)>d\left(x_{s}, x_{r}\right)$, transmission $\left(y_{s}, y_{r}\right)$ is successful.

Now we turn to UWB networks. According to inequality (1), in order to ensure a successful transmission $\left(x_{s}, x_{r}\right)$, the following inequality must hold.

$$
\frac{P_{x} / d\left(x_{s}, x_{r}\right)^{\alpha}}{N+P_{y} / d\left(y_{s}, x_{r}\right)^{\alpha}} \geq \frac{\beta}{m} \Rightarrow \frac{d\left(y_{s}, x_{r}\right)}{d\left(x_{s}, x_{r}\right)}>\left(\frac{\beta}{m}\right)^{\frac{1}{\alpha}} \cdot\left(\frac{P_{y}}{P_{x}}\right)^{\frac{1}{\alpha}}
$$

c) Protocol interference model with constant power assignment in UWB networks:

With the constant power assignment, similar to part a), by inequality (5) we have

$$
d\left(y_{s}, x_{r}\right)>(\beta / m)^{1 / \alpha} \cdot d\left(x_{s}, x_{r}\right)
$$

The interference range $\beta^{1 / \alpha} \cdot d\left(x_{s}, x_{r}\right)$ around the receiver $x_{r}$ is replaced with $(\beta / m)^{1 / \alpha} \cdot d\left(x_{s}, x_{r}\right)$. Hence the interference range becomes smaller than the transmission range.

For example, in Fig. 1(a), if $\alpha=4, \beta=2, m=100$, since $d\left(x_{s}, y_{r}\right)=2>(\beta / m)^{1 / \alpha} \cdot d\left(y_{s}, y_{r}\right) \doteq 1.5$,

the previously unsuccessful transmission $\left(y_{s}, y_{r}\right)$ with constant power assignment in narrowband networks becomes successful in UWB networks. As a result, the two transmissions can be scheduled in parallel.

d) Protocol interference model with linear power assignment in UWB networks:

With the linear power assignment, similar to part b), by inequality (5) we have

$\frac{d\left(y_{s}, x_{r}\right)}{d\left(x_{s}, x_{r}\right)}>\left(\frac{\beta}{m}\right)^{\frac{1}{\alpha}} \cdot \frac{d\left(y_{s}, y_{r}\right)}{d\left(x_{s}, x_{r}\right)} \Rightarrow d\left(y_{s}, x_{r}\right)>\left(\frac{\beta}{m}\right)^{\frac{1}{\alpha}} \cdot d\left(y_{s}, y_{r}\right)$

The interference range around receiver $x_{r}$ is changed from $(\beta / m)^{1 / \alpha} \cdot d\left(x_{s}, x_{r}\right)$ to $(\beta / m)^{1 / \alpha} \cdot d\left(y_{s}, y_{r}\right)$. For example, in Fig. 1(a), if $\alpha=4, \beta=2, m=100$, since

$d\left(y_{s}, x_{r}\right)=3>(\beta / m)^{1 / \alpha} \cdot d\left(y_{s}, y_{r}\right) \doteq 1.5$, the previously unsuccessful transmission $\left(x_{s}, x_{r}\right)$ with linear power assignment 
in narrowband networks becomes successful in UWB networks. So the two transmissions can be simultaneously scheduled.

From the above analyses, on one hand, due to the large processing gain $m$ when using the constant or linear power assignment, many unsuccessful simultaneous transmissions in narrowband networks become successful in UWB networks, thus leading to increased spatial reuse in UWB networks. On the other hand, as the examples in [16] have shown, even in narrowband networks, the unsuccessful simultaneous transmissions can also become successful with a proper arbitrary power assignment. For example, for Fig. 1(a), if $\alpha=4, \beta=2, N=1$, and $P_{x}=80, P_{y}=3150$, the two transmissions can be successfully scheduled in parallel. And for Fig. 1(c), if $\alpha=3, \beta=4, N=1$, and $P_{x}=14, P_{y}=64$, the two transmissions can also take place simultaneously. Nevertheless, in Section 4, we will show that even in UWB networks, both the constant and linear power assignments are still inefficient in terms of scheduling complexity. The following two theorems show that the power control technique has its limitations in reducing the scheduling complexity.

\subsection{Limitations of Power Control in Improving Spatial Reuse}

THEOREM 3.1. In narrowband networks, for any two transmissions $\left(x_{s}, x_{r}\right)$ and $\left(y_{s}, y_{r}\right)$, if $d\left(x_{s}, y_{r}\right) \cdot d\left(y_{s}, x_{r}\right) \leq$ $\beta^{2 / \alpha} \cdot d\left(x_{s}, x_{r}\right) \cdot d\left(y_{s}, y_{r}\right)$, then there exists no feasible power assignment for simultaneous transmissions; otherwise, there always exists a feasible power assignment to have a simultaneous schedule.

PROOF. If the two transmissions can be successfully scheduled, based on inequality (1) with processing gain equal to 1 , the following two inequalities must follow:

$$
\frac{P_{x} / d\left(x_{s}, x_{r}\right)^{\alpha}}{N+P_{y} / d\left(y_{s}, x_{r}\right)^{\alpha}} \geq \beta \quad \frac{P_{y} / d\left(y_{s}, y_{r}\right)^{\alpha}}{N+P_{x} / d\left(x_{s}, y_{r}\right)^{\alpha}} \geq \beta
$$

From these inequalities, we have

$$
\beta \cdot P_{y} \frac{d\left(x_{s}, x_{r}\right)^{\alpha}}{d\left(y_{s}, x_{r}\right)^{\alpha}}<P_{x}<\frac{1}{\beta} \cdot P_{y} \cdot \frac{d\left(x_{s}, y_{r}\right)^{\alpha}}{d\left(y_{s}, y_{r}\right)^{\alpha}}
$$

Therefore, if $\beta \cdot \frac{d\left(x_{s}, x_{r}\right)^{\alpha}}{d\left(y_{s}, x_{r}\right)^{\alpha}} \geq \frac{1}{\beta} \cdot \frac{d\left(x_{s}, y_{r}\right)^{\alpha}}{d\left(y_{s}, y_{r}\right)^{\alpha}}$, there is no feasible power assignment for simultaneous scheduling; otherwise, there always exists a feasible power assignment to schedule these two transmissions in parallel.

Actually this is a special case of the Perron-Frobenius theory used in the power control of cellular networks [9], but it has been paid little attention in multi-hop networks.

For example, in Fig. 1(d), if $\alpha=4, \beta=2$, and $N=1$, there will be no feasible power assignment to simultaneously schedule transmission $\left(x_{s}, x_{r}\right)$ and $\left(y_{s}, y_{r}\right)$. The same is true of Fig. 1(b).

THEOREM 3.2. In UWB (or any spread-spectrum) networks, for any two transmissions $\left(x_{s}, x_{r}\right)$ and $\left(y_{s}, y_{r}\right)$, if $d\left(x_{s}, y_{r}\right) \cdot d\left(y_{s}, x_{r}\right)>(\beta / m)^{2 / \alpha} \cdot d\left(x_{s}, x_{r}\right) \cdot d\left(y_{s}, y_{r}\right)$, there always exists a power assignment to schedule these transmissions in parallel; no feasible power assignments for simultaneous schedule, otherwise.

PROOF. Similar to the proof of Theorem 3.1, if the two transmissions can be successfully scheduled, the following two inequalities must follow:

$$
\frac{P_{x} / d\left(x_{s}, x_{r}\right)^{\alpha}}{N+P_{y} / d\left(y_{s}, x_{r}\right)^{\alpha}} \geq \frac{\beta}{m} \quad \frac{P_{y} / d\left(y_{s}, y_{r}\right)^{\alpha}}{N+P_{x} / d\left(x_{s}, y_{r}\right)^{\alpha}} \geq \frac{\beta}{m}
$$

From these inequalities, we have

$$
\frac{\beta}{m} \cdot P_{y} \frac{d\left(x_{s}, x_{r}\right)^{\alpha}}{d\left(y_{s}, x_{r}\right)^{\alpha}}<P_{x}<\frac{m}{\beta} \cdot P_{y} \cdot \frac{d\left(x_{s}, y_{r}\right)^{\alpha}}{d\left(y_{s}, y_{r}\right)^{\alpha}}
$$

Therefore, if $\frac{\beta}{m} \cdot \frac{d\left(x_{s}, x_{r}\right)^{\alpha}}{d\left(y_{s}, x_{r}\right)^{\alpha}}<\frac{m}{\beta} \cdot \frac{d\left(x_{s}, y_{r}\right)^{\alpha}}{d\left(y_{s}, y_{r}\right)^{\alpha}}$, there always exists a power assignment to simultaneously schedule these two transmissions; otherwise, there is no valid power assignment to give a parallel schedule.

For example, in Fig. 1(d), if $\alpha=4, \beta=2, \mathrm{~N}=1$, and $\mathrm{m}=10$, $P_{x}=P_{y}=1000$, the two transmissions can be simultaneously scheduled.

Therefore, given any two transmissions in narrowband networks where power control cannot guarantee a simultaneous schedule, they can be scheduled in parallel in UWB networks as long as $d\left(x_{s}, y_{r}\right) \cdot d\left(y_{s}, x_{r}\right)>(\beta / m)^{2 / \alpha} \cdot d\left(x_{s}, x_{r}\right) \cdot d\left(y_{s}, y_{r}\right)$. Given this result, we will discuss the scheduling complexity problem in UWB networks in Section 5.

\section{SCHEDULING COMPLEXITY AND INEFFICIENCY OF CONSTANT AND LINEAR POWER ASSIGNMENTS IN UWB NETWORKS}

\subsection{Scheduling Complexity}

We consider an arbitrarily distributed network with nodes $X=\left\{x_{0}, x_{1}, \ldots, x_{n-1}\right\}$ in the Euclidean plane. For any links $f_{i j}=\left(x_{i}, x_{j}\right), \ell\left(f_{i j}\right)=d\left(x_{i}, x_{j}\right)$ denotes the distance between node $x_{i}$ and node $x_{j}$.

DEFINITION 4.1. A power assignment $\phi_{t}$ is a function $\phi_{t}: X \rightarrow \mathbb{R}^{+}$which maps every node in the network to a certain power level. $\phi_{t}\left(x_{i}\right)=P_{i}$ denotes the power level of node $x_{i}$ in timeslot $t$. A schedule $S=\left(\phi_{1}, \phi_{2}, \ldots, \phi_{T(S)}\right)$ is a sequence of $T(S)$ power assignments, where $\phi_{i}$ denotes the power assignment in timeslot $i$.

DEFINITION 4.2. Given a timeslot $t$ and a power assignment $\phi_{t}$, we say that the directed link $\left(x_{s}, x_{r}\right)$ is successfully scheduled in timeslot $t$ if $x_{r}$ receives a message from $x_{s}$ according to the SINR inequality (1).

Let $E_{t}$ be the set of all successful links in timeslot $t$, we have DEFINITION 4.3. The scheduling problem for a network property $\Psi$ is to find a schedule $S$ of minimal length $T(S)$, such that the union of the set $E_{t}\left(\bigcup_{t=1}^{T(S)} E_{t}\right)$ satisfies the property $\Psi$.

DEFINITION 4.4. The scheduling complexity of a network property $\Psi$ is the minimal number of timeslots $T$, such that there always exists a valid schedule $S$ for $\Psi$ of length $T=T(S)$.

\subsection{The Inefficiency of Constant and Linear Power Assignments in UWB Networks}

We first define the following property $\psi_{\min }$ which is the same as that in [10]: For every node $x_{i} \in X$, it can successfully send at least one message to any other node. This is the simplest property to check the algorithm's scheduling complexity. We also consider the exponential node chain [7], where all the nodes are placed on a straight line with exponentially increasing distances between them. Fig. 2 below is an example.

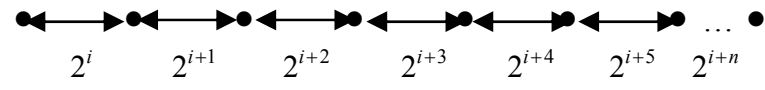

Figure 2: Exponential node chain, where $2^{i+n}$ is the distance between nodes $x_{n}$ and $x_{n+1}$ 
THEOREM 4.1. For both constant and linear power assignments, the scheduling complexity for problem $\psi_{\min }$ is at least $n \cdot \beta /\left(m \cdot 2^{\alpha}+\beta\right) \in \Omega(n / m)$, even in the absence of ambient noise, where $n$ is the number of the nodes, and $m$ is the processing gain.

\section{PROOF. a) Constant power assignment:}

In this case, for all nodes, transmission power $P_{i}=P_{k}=\mathrm{P}$. Now consider the example in Fig. 2; we assume there are at most $L$ simultaneous transmissions in a scheduling timeslot. Suppose node $x_{s}$ is the right-most transmitter in this timeslot, and node $x_{r}$ is its receiver. The other $(L-1)$ simultaneous transmissions will cause aggregate interferences to node $x_{r}$. According to the property of the exponential node chain, if node $x_{r}$ is on the left side of node $x_{s}$, the distance from every other simultaneous transmitter to the receiver $x_{r}$ is $d\left(x_{i}, x_{r}\right) \leq d\left(x_{s}, x_{r}\right)$; and if node $x_{r}$ is on the right side of node $x_{s}$, the distance from every other simultaneous transmitter to the receiver $x_{r}$ is $d\left(x_{i}, x_{r}\right) \leq 2 \cdot d\left(x_{s}, x_{r}\right)$. Therefore the aggregate interference caused by these $(L-1)$ simultaneous transmitters is at least $(L-1) \cdot P /\left(2 \cdot d\left(x_{s}, x_{r}\right)\right)^{\alpha}$. According to the SINR inequality (1), we have:

$\frac{P / d\left(x_{s}, x_{r}\right)^{\alpha}}{N+(L-1) \cdot P /\left(2 \cdot d\left(x_{s}, x_{r}\right)\right)^{\alpha}} \geq \frac{\beta}{m}$

From this, it follows that the maximum number of simultaneous transmissions $L$ in each timeslot is $\left(\beta+m \cdot 2^{\alpha}\right) / \beta$. Therefore, the constant power assignment method requires at least $n \cdot \beta /\left(\beta+m \cdot 2^{\alpha}\right)$ timeslots to schedule all nodes at least once.

\section{b) Linear power assignment:}

With linear power assignment, the sender $x_{s}$ will send to its receiver $x_{r}$ with power $P_{s}=\rho \cdot d\left(x_{s}, x_{r}\right)^{\alpha}$, where $\rho$ denotes the minimum received power to decode the message. Similar to the constant power assignment analysis, we assume there are at most $L$ simultaneous transmissions in a scheduling timeslot. According to the property of the exponential node chain, for all nodes $x_{i}$, it will cause at least the interference $\rho / 2^{\alpha}$ to its left side nodes [16]. Now suppose $x_{r}$ is the left-most receiver, and $x_{s}$ is some transmitter in the $L$ simultaneous transmissions. The other $(L-1)$ simultaneous transmissions will cause at least the aggregate interference $(L-1) \cdot \rho / 2^{\alpha}$ to this left-most receiver $x_{r}$. According to the SINR inequality (1), we have

$$
\frac{\rho \cdot d\left(x_{s}, x_{r}\right)^{\alpha} / d\left(x_{s}, x_{r}\right)^{\alpha}}{N+(L-1) \cdot \rho / 2^{\alpha}} \geq \frac{\beta}{m}
$$

From this, it follows that the maximum number of simultaneous transmissions $L$ in each timeslot is $\left(\beta+m \cdot 2^{\alpha}\right) / \beta$. And therefore the linear power assignment method requires also at least $n \cdot \beta /\left(\beta+m \cdot 2^{\alpha}\right)$ timeslots to schedule all nodes at least once.

\section{THE SCHEDULING COMPLEXITY OF STRONG CONNECTIVITY IN UWB NETWORKS}

\subsection{The NPAW Scheduling Algorithm}

It is important to distinguish between link length class and link length class set which are used in our algorithm. A link length class is a set of transmission links such that the lengths of these links differ by at most a factor of 2 (line 6 of the main algorithm). A number of link length classes form a link length class set. The three kinds of link length class set $L, S$ and $I$ used in our algorithm, and their relationships, are described in Fig. 3.

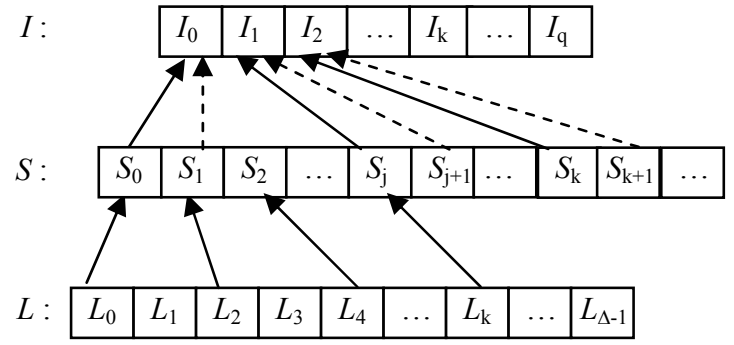

Figure 3: Three kinds of link length class set and their relationships.

In Fig. $3, L_{\mathrm{k}}, S_{\mathrm{k}}$ and $I_{\mathrm{k}}$ denote the respective length classes in each set. $L$ is renamed to $S$ because the empty length classes (containing no transmission links) in $L$ were deleted (line 7 of the main algorithm). For example, the length classes $L_{1}$ and $L_{3}$ were deleted. $S$ is renamed to $I$ because in each round, the scheduling algorithm only selects the length classes in $S$ with a certain length class separation. The separation value is $\log (4 \beta n)$ in [10] but we use $\log (3 n \beta / m)$ in our algorithm (line 9 of the main algorithm). The solid arrows from $S$ to $I$ mean we select the length classes $S_{0} S_{\mathrm{j}} S_{\mathrm{k}} \ldots$ in the first round, while the dashed arrows mean we select the length classes $S_{1} S_{\mathrm{j}+1} S_{\mathrm{k}+1} \ldots$ in the second round (the details are in Tables 1 and 2). Note that only links in $L_{\mathrm{k}}$ have the property $2^{k} \leq \ell\left(f_{i j}\right)<2^{k+1}$, but not those in $S_{\mathrm{k}}$ or $I_{\mathrm{k}}$ (because $2^{k+1}$ upper bound would not hold for them).

Our scheduling algorithm also uses a non-linear power assignment. For convenience, we refer to the scheduling algorithm in [10] as "NPAN" (non-linear power assignment for narrowband networks), and our algorithm "NPAW" (non-linear power assignment for (ultra)-wideband networks). The main algorithm of NPAW proceeds in phases. In each phase, the algorithm constructs a directed nearest neighbor forest $F_{p}$ on the current active nodes (lines 3, 4 and 5). Initially all the nodes in $X$ are active, but after every directed link in $F_{p}$ has been scheduled, all the transmitting nodes of these links become passive in the next phase (line 11). When there is only one active node, the while loop (line 2) terminates and the node set $X$ becomes a directed spanning tree with the active node as the sink (A sink node means a node having no outgoing link). Then the sink node transmits with maximum power to cover all the nodes in $X$ (line 13), so that the resultant topology would become strongly connected.

The challenging part of the algorithm is how to schedule $F_{p}$ both successfully and efficiently. Just as Fig. 3 has demonstrated, we first partition all the links in $F_{p}$ into length classes of $L$ which is then renamed to $S$ (lines 6 and 7). Then we use the subroutine Schedule() to schedule the links in length classes $S_{h \cdot \log (3 n \beta / m)+k}$ in the $k^{\text {th }}$ round (lines 8,9 and Table 2). The trick of this algorithm lies in two aspects: one is the non-linear power assignment scheme (line 9 of the subroutine). This power assignment uses a power scaling factor $\tau$ which depends on the position of the scheduling links in link length class set $I$ (lines 1 and 2 of the subroutine and Fig. 3). Because short links have a high $\tau$ value and long links have a low $\tau$ value, this power assignment can increase the power of the short links relative to the long ones so that it makes simultaneous transmissions of very different lengths possible. Furthermore, because this power assignment takes the parameter $n$ (total number of the nodes) into account, it can bound the aggregate interferences through the properly designed protocol interference model (lines 12 and 13 of 
the subroutine). But as discussed in Sections 2 and 3.1, traditional pair-wise protocol interference models cannot guarantee the successful transmission due to the aggregate interference effect.

The second part of the trick is the selection of the simultaneous transmitting links in length class set $I$ (Fig. 3). With the proper length class separation, the algorithm can bound the total number of deleting links at $O(\log n)$ in each timeslot of every scheduling round (line 9 of the main algorithm and lines 12 and 13 of the subroutine), thus guaranteeing that at least $\Omega(1 / \log n)$ of the current candidate links can be simultaneously transmitted in a single timeslot. Therefore the polylogarithmic scheduling complexity can be arrived at.

There are three important differences of our NPAW algorithm from the NPAN algorithm:

a) We design a new non-linear power assignment for UWB networks (line 9 of the subroutine).

b) We design two new protocol interference models which can bound the aggregate interferences through deleting fewer or at most equal transmitting links than the NPAN algorithm. The deleted links consist of two groups: the first are in the same length class link scheduling (line 1 of the main algorithm, and line 12 of the subroutine) and the second are in different length class link schedulings (line 13 of the subroutine). The proofs will be given in Corollary 5.5 and Lemma 5.6.

c) As shown in Fig. 3 and Table 2, there are more length classes to be scheduled in the same round in link length class set $I$ (line 9 of the main algorithm), and therefore the total iterations of the "for" loop is reduced as compared to the NPAN algorithm (line 8 of the main algorithm). By this reduction, we reduce the scheduling complexity from $O\left(\log ^{4} n\right)$ to $O\left(\log (n / m) \cdot \log ^{3} n\right)$.

Main Algorithm: Strong Connectivity Scheduling Algorithm for UWB Networks (NPAW)

Input: An arbitrarily distributed set of nodes $X$ (c.f. section 4.1)

Output: A schedule $S$ satisfying strong-connectivity

1: Define a constant $v:=4 N$ and a variable $\mu$ which is a function of the processing gain $m$ such that

$\mu:=2+\varepsilon+4 \cdot(72)^{\frac{1}{\alpha}} \cdot \sqrt[\alpha]{\frac{\beta}{m} \frac{(\alpha-1)}{(\alpha-2)}} ; \quad \alpha>2 ; t:=1 ;$

$\{N$ is the background noise from inequality (1) and $\varepsilon$ is a small positive parameter.

2: while $|X|>1$ do

3: $F_{p}:=\varnothing$;

4: For each $x_{i} \in X$ find its closest neighbor $x_{j}$ such that $F_{p}:=F_{p} \cup f_{i j} ; \quad\left\{f_{i j}\right.$ is a directed edge from $x_{i}$ to $\left.x_{j} \cdot\right\}$

5: If $F_{p}$ contains bi-directional edges then remove one edge of them; \{ To make $F_{n}$ a directed nearest neighbor forest. \}

6: Partition all the transmission links in $F_{p}$ into length class set $L=\left\{L_{0}, L_{1}, \ldots, L_{\Delta-1}\right\}$, such that $L_{k}$ contains all links $f_{i j}$ of length $2^{k} \leq \ell\left(f_{i j}\right)<2^{k+1} ;\left\{\Delta=\left\lceil\log \left(l_{\max }\right)\right\rceil\right.$, and $l_{\max }$ means the maximum link length in $F_{p}$.

7: Delete all empty length classes $L_{k}$ in $F_{p}$ and rename $L$ to $S=\left\{S_{0}, S_{1}, \ldots, S_{k}, \ldots\right\}$ such that $S_{k}$ is the $k^{\text {th }}$ smallest non-empty length-class in $S$;

8: $\quad$ for $k=0$ to $\log (3 n \beta / m)-1$ do

9: $\quad$ Schedule all the links

$$
f_{i j} \in \bigcup_{h=0}^{n / \log (3 n \beta / m)-1} S_{h \cdot \log (3 n \beta / m)+k}
$$

using subroutine Schedule();
10: $\quad$ end for

11: Delete all the nodes from node set $X$ except the sink node in each tree of the directed nearest neighbor forest $F_{p}$;

12: end while

13: $\phi_{t}\left(x_{i}\right):=N / m \cdot \beta \cdot l_{\max }^{\alpha}$ for $x_{i} \in X ;\left\{\right.$ Here $l_{\max }$ means the largest Euclidean distance between two nodes in node set $X$.

14: $S:=\left\{\phi_{1}, \ldots, \phi_{t-1}\right\}$;

Subroutine Schedule():

1: Let $F$ be the set of links to be scheduled, rename these link length classes in $S$ to $I=\left\{I_{0}, I_{1}, \ldots, I_{q}\right\}$ with at most $q+1$ length classes where $q=\lceil n / \log (3 n \beta / m)-1\rceil . I_{k}$ is the $k^{\text {th }}$ smallest length-class in $I$; \{line 9 of the main algorithm

2: for each $f_{u v} \in I_{k}$ do $\tau\left(x_{u}\right):=q-k+1$; \{Links within the smallest length class $I_{0}$ have the highest $\tau$ value $\lceil n / \log (3 n \beta / m)\rceil$, and links within the largest length class $I_{q}$ have the lowest $\tau$ value 1.$\}$

3: while $F \neq \varnothing$ do

4: for each $x_{i} \in X$ do $\phi_{t}\left(x_{i}\right):=0$; end for $\quad\{$ Set the powers of the transmitters in the previously scheduled links to 0.$\}$

5: $\quad F_{t}:=F ; E_{t}:=\varnothing$;

6: $\quad$ while $F_{t} \neq \varnothing$ do

7: $\quad$ Choose the link $f_{i j}^{*} \in F_{t}$ of minimal length;

8: $\quad E_{t}:=E_{t} \cup\left\{f_{i j}^{*}\right\} ; F_{t}:=F_{t} \backslash\left\{f_{i j}^{*}\right\} ;$

9: $\quad \phi_{t}\left(x_{i}\right):=v(3 n \beta / m)^{\tau\left(x_{i}\right)} \cdot \ell\left(f_{i j}^{*}\right)^{\alpha} ; \quad$ Schedule link $\left.f_{i j}^{*}\right\}$

10: $\quad$ for each $f_{k l} \in F_{t}$ do

11: $\quad \delta_{i k}:=\tau\left(x_{i}\right)-\tau\left(x_{k}\right)$;

12: $\quad$ if $\delta_{i k}=0$ and $\mathrm{d}\left(x_{i}, x_{l}\right) \leq \mu \cdot \ell\left(f_{i j}^{*}\right)$

then $F_{t}:=F_{t} \backslash\left\{f_{k l}\right\}$;

13: $\quad$ else if $\mathrm{d}\left(x_{i}, x_{l}\right) \leq(3 n \beta / m)^{\left(\delta_{i k}+1\right) / \alpha} \cdot \ell\left(f_{i j}^{*}\right)$ then $F_{t}:=F_{t} \backslash\left\{f_{k l}\right\}$;

14: $\quad$ end if

15: $\quad$ end for

16: end while

17: $\quad F:=F \backslash E_{t} ; \quad t:=t+1$;

18: end while

\subsection{Correctness Analysis}

In Section 5.1, we have shown that in each timeslot of every scheduling round, the deleting links in the NPAW algorithm must be fewer than or at most equal to the deleting links in the NPAN algorithm. This means that there are more transmission links that should be scheduled in the same timeslot, thus proving that the successful simultaneous transmissions of all available links is of fundamental importance.

LEMMA 5.1. Consider a scheduled link $f_{x}$ with intended sender $x_{s}$ and receiver $x_{r}$. Let $I_{r}\left(y_{i}\right)$ be the interference caused at $x_{r}$ by simultaneously transmitting nodes $y_{i}$ for which $\tau\left(y_{i}\right)<\tau\left(x_{s}\right)$. It holds that $I_{r}\left(y_{i}\right) \leq v(3 n \beta / m)^{\tau\left(x_{s}\right)-1}$.

PROOF. In our main algorithm, because every node $y_{i}$ transmits messages to its nearest neighbor, we have $d\left(y_{i}, x_{r}\right) \geq \ell\left(f_{y}\right)$. Hence the interference at $x_{r}$ caused by $y_{i}$ is at most

$I_{r}\left(y_{i}\right)=$ 
$\frac{P_{i}}{d\left(y_{i}, x_{r}\right)^{\alpha}} \leq \frac{v(3 n \beta / m)^{\tau\left(y_{i}\right)} \cdot \ell\left(f_{y}\right)^{\alpha}}{\ell\left(f_{y}\right)^{\alpha}}=v\left(\frac{3 n \beta}{m}\right)^{\tau\left(y_{i}\right)} \leq v\left(\frac{3 n \beta}{m}\right)^{\tau\left(x_{s}\right)-1}$.

LEMMA 5.2. Consider a scheduled link $f_{x}$ with intended sender $x_{s}$ and receiver $x_{r}$. Let $I_{r}\left(y_{i}\right)$ be the interference caused at $x_{r}$ by simultaneously transmitting nodes $y_{i}$ for which $\tau\left(y_{i}\right)>\tau\left(x_{s}\right)$. It holds that $I_{r}\left(y_{i}\right) \leq v(3 n \beta / m)^{\tau\left(x_{s}\right)-1}$.

PROOF. Assume for contradiction that there exists a node $y_{i}$ with $\tau\left(y_{i}\right)>\tau\left(x_{s}\right)$ and $I_{r}\left(y_{i}\right)>v(3 n \beta / m)^{\tau\left(x_{s}\right)-1}$. Then

$$
I_{r}\left(y_{i}\right)=\frac{P_{i}}{d\left(y_{i}, x_{r}\right)^{\alpha}} \leq \frac{v(3 n \beta / m)^{\tau\left(y_{i}\right)} \cdot \ell\left(f_{y}\right)^{\alpha}}{d\left(y_{i}, x_{r}\right)^{\alpha}}>v\left(\frac{3 n \beta}{m}\right)^{\tau\left(x_{s}\right)-1}
$$

From this, we have $d\left(y_{i}, x_{r}\right)<(3 n \beta / m)^{\left(\delta_{i s}+1\right) / \alpha} \cdot \ell\left(f_{y}\right)$.

However, this contradicts the definition of our algorithm. In line 13 of the subroutine, if node $y_{i}$ has been scheduled (because it has short link length, line 7 of the subroutine), from the above inequality, node $x_{s}$ should have been deleted, which establishes the contradiction. Therefore, $I_{r}\left(y_{i}\right) \leq v(3 n \beta / m)^{\tau\left(x_{s}\right)-1}$ holds.

LEMMA 5.3. Consider a scheduled link $f_{x}$ with intended sender $x_{s}$ and receiver $x_{r}$. Let $I_{r}^{0}$ be the total interferences caused at $x_{r}$ by simultaneously transmitting nodes $y_{i}$ for which $\tau\left(y_{i}\right)=\tau\left(x_{s}\right)$. The following holds:

$$
I_{r}^{0} \leq(v / 3) \cdot(\beta / m)^{\tau\left(x_{s}\right)-1} \cdot(3 n)^{\tau\left(x_{s}\right)} \text {. }
$$

PROOF. The proof of this lemma is similar to that of Lemma 4.4 in [10]. The main idea is that because the lengths of the links in the same length class differ by at most a factor of 2 , according to a simple geometric area argument, the deleting links must be bounded by a certain number. The difference is that we change the ring width from $\frac{1}{2}(\mu-3) \cdot \ell\left(f_{x}\right)$ to $\frac{1}{2}(\mu-2-\varepsilon) \cdot \ell\left(f_{x}\right)$. And more importantly, the $\mu$ value is greatly reduced due to the introduction of the processing gain $m$ in the denominator. Thus the deleting links in the same length class are greatly reduced. Plugging in the value of $\mu$ in line 1 of the main algorithm, the results follow. We omit the detailed proof because of the lack of space.

THEOREM 5.4 For an arbitrary timeslot $t$, all scheduled transmissions $E_{t}$ in $t$ are received successfully by the intended receivers, and thus the computed schedule is correct.

PROOF. Consider a scheduled link $f_{x}$ with intended sender $x_{s}$ and receiver $x_{r}$. The aggregate interferences at this receiver $x_{r}$ can be calculated through Lemmas 5.1, 5.2 and 5.3.

By Lemmas 5.1 and 5.2, we know that for all $y_{i}$ with $\tau\left(y_{i}\right)>\tau\left(x_{s}\right)$ and $\tau\left(y_{i}\right)<\tau\left(x_{s}\right)$, the interference $I_{r}\left(y_{i}\right)$ is bounded by $v(3 n \beta / m)^{\tau\left(x_{s}\right)-1}$. Hence, because there are at most $n$ nodes in these sets, it holds that

$\sum_{y_{i}: \tau\left(x_{s}\right) \neq \tau\left(y_{i}\right)} I_{r}\left(y_{i}\right) \leq n \cdot v\left(\frac{3 n \beta}{m}\right)^{\tau\left(x_{s}\right)-1}=\frac{v}{3} \cdot\left(\frac{\beta}{m}\right)^{\tau\left(x_{s}\right)-1} \cdot(3 n)^{\tau\left(x_{s}\right)}$

Therefore the aggregate interference at $x_{r}$ is

$I_{r}=(v / 3) \cdot(\beta / m)^{\tau\left(x_{s}\right)-1} \cdot(3 n)^{\tau\left(x_{s}\right)}+(v / 3) \cdot(\beta / m)^{\tau\left(x_{s}\right)-1} \cdot(3 n)^{\tau\left(x_{s}\right)}$

$=2 \cdot(v / 3) \cdot(\beta / m)^{\tau\left(x_{s}\right)-1} \cdot(3 n)^{\tau\left(x_{s}\right)}$

And SINR at $x_{r}$ is

$\operatorname{SINR}=\frac{v \cdot(3 n \beta / m)^{\tau\left(x_{s}\right)} \cdot \ell\left(f_{x}\right)^{\alpha} / \ell\left(f_{x}\right)^{\alpha}}{N+2 \cdot(v / 3) \cdot(\beta / m)^{\tau\left(x_{s}\right)-1} \cdot(3 n)^{\tau\left(x_{s}\right)}}$

Since $v:=4 N$ (line 1 of the main algorithm)

$\operatorname{SINR}=\frac{v \cdot(3 n \beta / m)^{\tau\left(x_{s}\right)}}{N+2 \cdot(v / 3) \cdot(\beta / m)^{\tau\left(x_{s}\right)-1} \cdot(3 n)^{\tau\left(x_{s}\right)}} \geq \frac{\beta}{m}$
From this, we conclude that the computed schedule is correct.

\subsection{Efficiency Analysis}

COROLLARY 5.5. In each timeslot, the deleted links in the same length class in the NPAW algorithm are strictly fewer than the deleted links in the NPAN algorithm.

PROOF. This conclusion is from the proof of Lemma 5.3.

LEMMA 5.6. In each timeslot, the deleted links in different length classes in the NPAW algorithm are fewer than or at most equal to the deleted links in the NPAN algorithm.

PROOF. From line 13 of the subroutine, on one hand, if the difference of the power scaling factors between different length classes is the same, because we have introduced the processing gain $m$ as the denominator in the base, the deleted links must be fewer than its counterpart in NPAN. On the other hand, since $(3 n \beta / m)^{\left(\delta_{i k}+1\right) / \alpha} \leq(3 n \beta / m)^{(n / \log (3 n \beta / m)-1+1) / \alpha}=2^{n / \alpha}$, and since $(4 n \beta)^{\left(\delta_{i k}+1\right) / \alpha} \leq(4 n \beta)^{(n / \log (4 \beta n)-1+1) / \alpha}=2^{n / \alpha}$, the deleted links must be at most equal to its counterpart in NPAN.

THEOREM 5.7. The scheduling complexity for strong connectivity in UWB networks is $O\left(\log (n / m) \cdot \log ^{3} n\right)$.

PROOF. First of all, according to the construction of the directed nearest neighbor forest $F_{p}$ in each phase of the main algorithm, the number of the nodes in the forest $F_{p+1} \leq(1 / 2) \cdot F_{p}$. Hence at most $\log n$ directed nearest neighbor forests $F_{p}$ exist until there remains only a single active node (line 13 of the main algorithm). And according to Corollary 5.5 and Lemma 5.6, the total number of the deleting links in each timeslot of the same scheduling round must not exceed $O(\log n)$ which is the result of the NPAN algorithm. Hence, at least a fraction of $\Omega(1 / \log n)$ of the transmission links that remain to be scheduled in the $k^{\text {th }}$ scheduling round can be simultaneously scheduled in a single timeslot. Then after at most $x$ timeslots, the number of remaining nodes that still need to be scheduled is $y \cdot(1-1 / \log n)^{x} \leq y \cdot e^{-x / \log n}$, where $y$ is the initial number of nodes that need to be scheduled in $F_{p}(y \leq n)$. So when $x=\ln y \cdot \log n$, the number of remaining nodes in this scheduling round is 1 . Based on this observation, each scheduling round (the subroutine Schedule()) only requires at most $O\left(\log ^{2} n\right)$ timeslots. And according to line 8 of our main algorithm, there are at most $\log (3 n \beta / m)$ scheduling rounds; therefore the total scheduling complexity of this algorithm is:

$T(S) \leq O\left(\log ^{2} n\right) \cdot \log n \cdot \log (3 n \beta / m) \in O\left(\log (n / m) \cdot \log ^{3} n\right)$

\section{THE ENERGY COMPLEXITY OF WIRELESS SCHEDULING PROBLEMS}

We define the energy complexity of the wireless scheduling problem to be the total energy cost for successfully scheduling all the transmission requests to meet some topological property requirement.

THEOREM 6.1. For the strong connectivity scheduling algorithm in narrowband networks, the lower bound of the energy complexity is $\omega\left(n \cdot 2^{n}\right)$; and the upper bound of the energy complexity is $O\left(n^{2} \cdot 2^{n \alpha}\right)$, where $n$ is the number of the nodes.

PROOF. In the NPAN algorithm, only links in link length class $S_{h \cdot \log (4 \beta n)+k}$ can be scheduled simultaneously in the $k^{\text {th }}$ scheduling round ( $k$ is from 0 to $\log (4 \beta n)-1$, represented by the columns of Table 1). And $h$ is from 0 to $n / \log (4 \beta n)-1$ (represented by the rows of Table 1). In particular, let's consider the link length classes $S_{k}$ and $S_{n-\log (4 \beta n)+k}$, which are the shortest length class and the longest length class in the $k^{\text {th }}$ scheduling round, respectively. 
According to Fig. 3, suppose the length class $S_{k}$ is mapped from $L_{u}$,we have $u \geq k$; And suppose the length class $S_{n-\log (4 \beta n)+k}$ is mapped from $L_{n-\log (4 \beta n)+v}$, we have $v \geq u \geq k$. According to the power scaling factor $\tau$ of their algorithm, length class $S_{k}$ has the highest $\tau$ value $n / \log (4 \beta n)$; and length class $S_{n-\log (4 \beta n)+k}$ has the lowest $\tau$ value, of 1 . So according to the non-linear power assignment scheme in the algorithm, the power $P\left(S_{k}\right)$ assigned to the links in $S_{k}$ has the property

$v(4 \beta n)^{n / \log (4 \beta n)} \cdot 2^{u \alpha} \leq P\left(S_{k}\right)<v(4 \beta n)^{n / \log (4 \beta n)} \cdot 2^{(u+1) \alpha} \quad \Rightarrow$ $v \cdot 2^{n} \cdot 2^{u \alpha} \leq P\left(S_{k}\right)<v \cdot 2^{n} \cdot 2^{(u+1) \alpha}$

The power $P\left(S_{n-\log (4 \beta n)+k}\right)$ assigned to links in $S_{n-\log (4 \beta n)+k}$ has the property $P\left(S_{n-\log (4 \beta n)+k}\right) \geq v \cdot(4 \beta n)^{1} \cdot\left(2^{n-\log (4 \beta n)+v}\right)^{\alpha} \quad$ and

$P\left(S_{n-\log (4 \beta n)+k}\right)<v \cdot(4 \beta n)^{1} \cdot\left(2^{n-\log (4 \beta n)+v+1}\right)^{\alpha} \quad \Rightarrow$ $v \cdot 2^{n \alpha} \cdot 2^{v \alpha} /(4 \beta n)^{\alpha-1} \leq P\left(S_{n-\log (4 \beta n)+k}\right)<v \cdot 2^{n \alpha} \cdot 2^{(v+1) \alpha} /(4 \beta n)^{\alpha-1}$

Because $0 \leq k \leq u \leq v \leq \log (4 \beta n)-1$, we have $v \cdot 2^{n} \cdot 2^{0} \leq v \cdot 2^{n} \cdot 2^{u \alpha} \leq P\left(S_{k}\right)<v \cdot 2^{n} \cdot 2^{(u+1) \alpha} \leq$ $v \cdot 2^{n \alpha} \cdot 2^{v \alpha} /(4 \beta n)^{\alpha-1} \leq P\left(S_{n-\log (4 \beta n)+k}\right)<v \cdot 2^{n \alpha} \cdot 2^{(v+1) \alpha} /(4 \beta n)^{\alpha-1}$ $\leq v \cdot(4 \beta n) \cdot 2^{n \alpha}$

From this, and because the sink node of the final directed spanning tree transmits with the power $N \cdot \beta \cdot l_{\max }^{\alpha}$, which could be $N \cdot \beta \cdot 2^{n \alpha}$, we get the lower bound of the energy complexity for the strong connectivity scheduling problem in narrowband networks, which is $\omega\left(n \cdot 2^{n}\right)$, and the upper bound of the energy complexity, which is $O\left(n^{2} \cdot 2^{n \alpha}\right)$

THEOREM 6.2. For the strong connectivity scheduling algorithm in UWB (or any spread-spectrum) networks, the lower bound of the energy complexity is still $\omega\left(n \cdot 2^{n}\right)$; but the upper bound of the energy complexity is reduced to $O\left(\frac{1}{m} \cdot n^{2} \cdot 2^{n \alpha}\right)$, where $n$ is the number of the nodes and $m$ is the processing gain.

PROOF. With our main algorithm, only links in link length class $S_{h \cdot \log (3 n \beta / m)+k}$ can be scheduled simultaneously in the $k^{\text {th }}$ scheduling round ( $k$ is from 0 to $\log (3 n \beta / m)-1$, represented by the columns of Table 2); and $h$ is from 0 to $n / \log (3 n \beta / m)-1$ (represented by the rows of Table 2). In particular, let's consider the link length classes $S_{k}$ and $S_{n-\log (3 n \beta / m)+k}$, which are the shortest length class and the longest length class in the $k^{\text {th }}$ scheduling round, respectively.

According to Fig. 3, suppose the length class $S_{k}$ is mapped from $L_{u}$, we have $u \geq k$; and suppose the length class

$S_{n-\log (3 n \beta / m)+k}$ is mapped from $L_{n-\log (3 n \beta / m)+v}$, we have $v \geq u \geq k$. From line 2 of the subroutine Schedule(), the length class $S_{k}$ has the highest $\tau$ value $\lceil n / \log (3 n \beta / m)\rceil$, and the length class $S_{n-\log (3 n \beta / m)+k}$ has the lowest $\tau$ value, of 1 . So according to the non-linear power assignment scheme in our algorithm, the power $P\left(S_{k}\right)$ assigned to the links in $S_{k}$ has the property

$$
\begin{aligned}
& P\left(S_{k}\right) \geq v(3 n \beta / m)^{\lceil n / \log (3 n \beta / m)\rceil} \cdot 2^{u \alpha} \\
& P\left(S_{k}\right)<v(3 n \beta / m)^{\lceil n / \log (3 n \beta / m)\rceil} \cdot 2^{(u+1) \alpha} \\
& v \cdot 2^{n} \cdot 2^{u \alpha} \leq P\left(S_{k}\right)<v \cdot 2^{n} \cdot 2^{(u+1) \alpha}
\end{aligned}
$$$$
\text { and }
$$$$
\Rightarrow
$$

The power $P\left(S_{n-\log (3 n \beta / m)+k}\right)$ assigned to links in $S_{n-\log (3 n \beta / m)+k}$ has the property

$$
\begin{aligned}
& P\left(S_{n-\log (3 n \beta / m)+k}\right) \geq v \cdot(3 n \beta / m)^{1} \cdot\left(2^{n-\log (3 n \beta / m)+v}\right)^{\alpha} \quad \text { and } \\
& P\left(S_{n-\log (3 n \beta / m)+k}\right)<v \cdot(3 n \beta / m)^{1} \cdot\left(2^{n-\log (3 n \beta / m)+v+1}\right)^{\alpha} \quad \Rightarrow \\
& v \cdot 2^{(n+v) \alpha} /(3 n \beta / m)^{\alpha-1} \leq P\left(S_{n-\log (3 n \beta / m)+k}\right)<v \cdot 2^{(n+v+1) \alpha} /(3 n \beta / m)^{\alpha-1}
\end{aligned}
$$

Because $0 \leq k \leq u \leq v \leq \log (3 n \beta / m)-1$, we have $v \cdot 2^{n} \cdot 2^{0} \leq v \cdot 2^{n} \cdot 2^{u \alpha} \leq P\left(S_{k}\right)<v \cdot 2^{n} \cdot 2^{(u+1) \alpha} \leq$

$v \cdot 2^{(n+v) \alpha} /(3 n \beta / m)^{\alpha-1} \leq P\left(S_{n-\log (3 n \beta / m)+k}\right)<v \cdot 2^{(n+v+1) \alpha} /(3 n \beta / m)^{\alpha-1}$

$\leq v \cdot(3 n \beta / m) \cdot 2^{n \alpha}$

From this, and because the final sink node transmits with the power $N / m \cdot \beta \cdot l_{\max }^{\alpha}$ (line 13 of the main algorithm), which could be $N / m \cdot \beta \cdot 2^{n \alpha}$, we get the lower bound of the energy complexity for the strong connectivity scheduling problem in UWB networks is $\omega\left(n \cdot 2^{n}\right)$, and the upper bound of the energy complexity is $O\left(\frac{1}{m} \cdot n^{2} \cdot 2^{n \alpha}\right)$.

Table 1. Link length classes scheduling (in order) in narrowband networks (from left to right, from top to bottom).

\begin{tabular}{|l|l|l|l|l|}
\hline$S_{0}$ & $S_{\log (4 \beta n)}$ & $S_{2 \log (4 \beta n)}$ & $\ldots$ & $S_{n-\log (4 \beta n)}$ \\
\hline$S_{1}$ & $S_{\log (4 \beta n)+1}$ & $S_{2 \log (4 \beta n)+1}$ & $\ldots$ & $S_{n-\log (4 \beta n)+1}$ \\
\hline$\ldots$ & $\ldots$ & $\ldots$ & $\ldots$ & $\ldots$ \\
\hline$S_{k}$ & $S_{\log (4 \beta n)+k}$ & $S_{2 \log (4 \beta n)+k}$ & $\ldots$ & $S_{n-\log (4 \beta n)+k}$ \\
\hline$\ldots$ & $\ldots$ & $\ldots$ & $\ldots$ & $\ldots$ \\
\hline$S_{\log (4 \beta n)-1}$ & $S_{2 \log (4 \beta n)-1}$ & $S_{3 \log (4 \beta n)-1}$ & $\ldots$ & $S_{n-1}$ \\
\hline
\end{tabular}

Table 2. Link length classes scheduling in UWB networks (from left to right, from top to bottom).

\begin{tabular}{|l|l|l|l|l|}
\hline$S_{0}$ & $S_{\log (3 n \beta / m)}$ & $S_{2 \log (3 n \beta / m)}$ & $\ldots$ & $S_{n-\log (3 n \beta / m)}$ \\
\hline$S_{1}$ & $S_{\log (3 n \beta / m)+1}$ & $S_{2 \log (3 n \beta / m)+1}$ & $\ldots$ & $S_{n-\log (3 n \beta / m)+1}$ \\
\hline$\ldots$ & $\ldots$ & $\ldots$ & $\ldots$ & $\ldots$ \\
\hline$S_{k}$ & $S_{\log (3 n \beta / m)+k}$ & $S_{2 \log (3 n \beta / m)+k}$ & $\ldots$ & $S_{n-\log (3 n \beta / m)+k}$ \\
\hline$\ldots$ & $\ldots$ & $\ldots$ & $\ldots$ & $\ldots$ \\
\hline$S_{\log (3 n \beta / m)-1}$ & $S_{2 \log (3 n \beta / m)-1}$ & $S_{3 \log (3 n \beta / m)-1}$ & $\ldots$ & $S_{n-1}$ \\
\hline
\end{tabular}

From these two theorems, we can see that the polylogarithmic scheduling complexity comes at the expense of exponential energy complexity. The results can also be applied to the situation in [11] because they also adopt the NPAN algorithm. Compared with narrowband networks, by Theorem 6.2, we can see that the upper bound of the energy complexity can be reduced by a processing gain factor in UWB networks.

\section{CONCLUSIONS}

In this paper, we generalize the scheduling complexity in narrowband networks to UWB networks, and reduce it from $O\left(\log ^{4} n\right)$ to $O\left(\log (n / m) \cdot \log ^{3} n\right)$. By considering the impact of the arbitrary power assignment on pair-wise transmissions scheduling, we explicitly show that when some node distance function is satisfied, there does not exist any power assignment for simultaneous link scheduling, and thus the scheduling complexity cannot be further improved via the means of power assignment. Therefore, the scheduling algorithm must take full advantage of the power assignment schemes so that it can simultaneously schedule as many links as possible without violating the physical SINR model. Compared to narrowband networks, we show that there is more room for UWB networks to take full advantage of power control to improve the scheduling complexity. Besides the power control limitations, we also give a detailed analysis on the power control impact on the pair-wise protocol interference models. More importantly, we explicitly prove that the polylogarithmic scheduling complexity is gained at the expense of exponential energy complexity in both narrowband networks and UWB networks.

In order to turn our algorithm into a practical network protocol, some problems need to be solved first, including the following.

1) Although in UWB networks, the upper bound of the energy complexity can be reduced by a processing gain factor, the 
exponential energy complexity lower bound would not change. Thus reducing the energy complexity without sacrificing the scheduling complexity is a very interesting and challenging task. To take up this challenge, a new arbitrary power assignment may need to be designed.

2) Our paper assumes that the traffic demand of all links is 1, but in some realistic wireless networks, different links experience different traffic demands. For example, the nodes near the wireless router in wireless mesh networks experience more traffic demands than the nodes on the border of the network [3]. Therefore, devising some efficient link scheduling to meet both topological requirements and arbitrary link traffic demands is another very challenging and interesting problem. Constraintprogramming techniques may be useful.

3) With the non-linear power assignment, every transmitting node must know its own power scaling factor $\tau$, which is based on some global picture, thus making it difficult to implement the algorithm in a distributed manner. To take up this challenge, implementing a clustering algorithm is a possible method.

4) Our algorithm assumes one channel is used, but actually in MIMO networks (e.g., 802.11n), a node can be equipped with multiple radios and operate on multiple channels. Thus extending our algorithm to multi-radio multi-channel scenarios is a natural idea.

\section{ACKNOWLEDGMENTS}

We would like to thank Prof. Roger Wattenhofer and Dr. Thomas Moscibroda for their valuable suggestions. We also thank the anonymous reviewers for their comments to improve the presentation of this paper.

\section{REFERENCES}

[1] H. Balakrishnan, C. Barrett, V. S. Anil Kumar, M. Marathe, and $\mathrm{S}$. Thite. The distance 2-matching problem and its relationship to the MAC layer capacity of ad-hoc wireless networks. IEEE J. Selected Areas in Communications, 22(6):1069-1079, August 2004.

[2] A. Behzad and I. Rubin. On the performance of graph-based scheduling algorithms for packet radio networks. In Proc. of IEEE Global Telecommunications Conference (GLOBECOM), 6:3432-3436, San Francisco, December 2003.

[3] G. Brar, D. Blough, and P. Santi. Computationally efficient scheduling with the physical interference model for throughput improvement in wireless mesh networks. In Proc. Twelfth ACM Annual International Conference on Mobile Computing and Networking (MOBICOM), Los Angeles, CA, US, Sept. 2006.

[4] F. Cuomo, C. Martello, A. Baiocchi and F. Capriotti. Radio resource sharing for ad-hoc networking with UWB. IEEE Journal on Selected Areas in Communications, 20(9):17221732, December 2002.

[5] M. Grossglauser and D. Tse. Mobility increases the capacity of ad-hoc wireless networks. IEEE/ACM Transactions on Networking, 10(4):477-486, August, 2002.
[6] Piyush Gupta and P. R. Kumar. The capacity of wireless networks. IEEE Transactions on Information Theory, 46(2):388-404, 2000.

[7] F. Meyer auf der Heide, C. Schindelhauer, K. Volbert, and M. Gruenewald. Energy, congestion and dilation in radio networks. In Proc. of the 14th Annual ACM Symp. on Parallel Algorithms and Architectures (SPAA), Winnipeg, Canada, August 2002.

[8] K. Jain, J. Padhye, V. N. Padmanabhan, L. Qiu. Impact of interference on multi-hop wireless network performance. Wireless Networks, 11(4): 471-487, 2005.

[9] S. Koskie and Z. Gajic. Signal-to-interference-based power control for wireless networks: a survey, 1992-2005.

Dynamics of Continuous, Discrete and Impulsive Systems B: Applications and Algorithms, 13(2):187-220, 2006.

[10] T. Moscibroda and R. Wattenhofer. The complexity of connectivity in wireless networks. In Proc. 25th Annual Joint Conference of the IEEE Computer and Communications Societies (INFOCOM), Barcelona, Spain, April 2006.

[11] T. Moscibroda, R. Wattenhofer, and A. Zollinger. Topology control meets SINR: the scheduling complexity of arbitrary topologies. In Proc. 7th ACM International Symposium on Mobile Ad Hoc Networking and Computing (MOBIHOC), Florence, Italy, May 2006.

[12] R.C. Qiu, H. Liu, and X. Shen. Ultra-wideband for multipleaccess communications. IEEE Communications Magazine, 43(2):80-87, Feb. 2005.

[13] B. Radunovic and J.-Y. Le Boudec. Optimal power control, scheduling and routing in UWB Networks. IEEE Journal on Selected Areas in Communications, 22(7):1252-1270, Sept. 2004.

[14] S. Schmid and R. Wattenhofer. Algorithmic models for sensor networks. In Proc. 14th International Workshop on Parallel and Distributed Real-Time Systems (WPDRTS), Island of Rhodes, Greece, April 2006.

[15] A. Sen and M. L. Huson. A new model for scheduling packet radio networks. Wireless Networks, 3:71-82, 1997.

[16] R. Wattenhofer. MACbeth: The three witches of media access theory . In 1st IEEE International Workshop on Foundation and Algorithms for Wireless Networking $(F A W N)$, Pisa, Italy, March 2006.

[17] M. Z. Win and R. A. Scholtz. Ultra-wide bandwidth timehopping spread-spectrum impulse radio for wireless multiple-access communications. IEEE Transactions on Communications, 48(4):679-691,2000

[18] X. Yang and G. de Veciana. Inducing spatial clustering in MAC contention for spread spectrum ad hoc networks. In Proc. 6th ACM International Symposium on Mobile Ad Hoc Networking and Computing (MOBIHOC), UrbanaChampaign, IL, USA May 2005. 